\title{
Minimizing the Use of Polyethene inside Paper Coffee Cups
}

\section{Romina Mahinpei}

University of Brtish Columbia

ABSTRACT: Although made of paper, most coffee cups are not recycled because of the polyethene covering their internal surface area $(1,2)$. Instead, they are sent to landfills where they break down into microplastics and negatively impact organisms after entering the food chain $(2,3)$. This is an especially alarming issue due to the extensive usage of paper coffee cups around the world. As a result, many global companies have been searching for an eco-friendly cup that eliminates the use of polyethene, a challenge that remains unresolved to this day (9). While the search continues, many businesses have relied on temporary strategies to reduce polyethene production until a design that eliminates its use is developed $(9,10,11,12)$. Two major methods include public awareness and promotion of reusable cups (2). However, these approaches have only resulted in minor changes due to their reliance on customer cooperation (2).

To guarantee polyethene reduction, this report proposes a strategy that is independent of customer cooperation. This method determines the dimensions (i.e., height and bottom radius) that minimize the amount of polyethene needed to coat the internal surface area of a cup, while keeping the cup's volume and lid size (i.e., top radius) the same. The resulting equation gives the surface area of the cup while the root to the first derivate of this equation corresponds to the optimal bottom radius. Using a derived equation for height, the optimal cup height is determined as well. To highlight its proper implementation, this strategy is applied to a Starbucks Grande coffee cup as a model for other companies to follow.

KEYWORDS: Polyethene, paper coffee cups, environmental stewardship

\section{INTRODUCTION}

Although advertised as 'recyclable', less than $1 \%$ of paper coffee cups are recycled due to the polyethene coating covering the internal surface area of these cups (1). The strong adhesion between the polyethene coating and the paper cup prevents standard recycling facilities from separating the two components (2). As a result, the majority of paper coffee cups are sent directly to landfills, where they take over 20 years to decompose while also releasing methane into the atmosphere (2). In addition, the gradual breakdown of polyethene produces microplastics that can enter the food chain via ingestion and negatively impact the health of organisms, including humans (3).

Numerous strategies have been developed in response to the negative impacts associated with paper coffee cups $(8,9,10$, 11). Major approaches include public awareness and promotion of reusable cups as companies around the world search for a fully recyclable design. Nonetheless, the search continues just as the global usage of paper coffee cups continues to grow every day. This is because of the reliance of these methods on customer cooperation and behavioural change, which are not only out of the control of companies but also difficult to modify. As explained in the Fogg Model of Behaviour Change, motivation is a necessity for behavioural change (4). However, for many customers, comfort is a stronger motivator than environmental sustainability, thus limiting the success of the current approaches.

As a way to minimize the inevitable production of polyethene without relying on consumers, this paper provides an approach to finding the optimal dimensions that minimize the surface area of polyethene inside a coffee cup. Using this approach, companies can redesign their cups to minimize polyethene production, as is shown with a Starbucks Grande coffee cup.

\section{METHODOLOGY}

To offer the same quantity of a beverage and minimize the redevelopment costs for companies, this approach treats the cup's volume and lid size (i.e., the top radius) as constants and 
keeps the geometry of the paper cup as a conical frustum, a geometric shape made by cutting off the top of a cone. In doing so, this approach provides the dimensions for the height and bottom radius of a cup that minimizes the amount of polyethene for a given volume and top radius.

Using the equation for the volume of a conical frustum as derived in Appendix $A$, the height of the cup is expressed in terms of the bottom radius. Note that the volume and top radius are not variables but constants that are determined using the original dimensions of the chosen cup.

$$
\begin{gathered}
V=\frac{\pi h}{3}\left(r^{2}+R r+R^{2}\right) \\
\boldsymbol{h}=\frac{3 \boldsymbol{V}}{\pi\left(\boldsymbol{r}^{2}+\boldsymbol{R} \boldsymbol{r}+\boldsymbol{R}^{2}\right)}
\end{gathered}
$$

(Appendix A)

where:

$$
\begin{array}{ll}
V=\text { volume of cup } & r=\text { bottom radius of cup } \\
R=\text { top radius of cup } & h=\text { height of cup }
\end{array}
$$

Next, equation 1.1 is substituted for $h$ in the equation for the internal surface area of a conical frustum as derived in Appendix B, which provides an internal surface area equation that is expressed in terms of one variable (i.e., the bottom radius).

$$
A=\pi r^{2}+\pi(r+R) \sqrt{(r-R)^{2}+h^{2}}
$$

(Appendix B)

$$
A=\pi r^{2}+\pi(r+R) \sqrt{(r-R)^{2}+\left(\frac{3 V}{\pi\left(r^{2}+R r+R^{2}\right)}\right)^{2}}
$$

Then, the root to the first derivative of equation 1.2 is used to determine the dimensions that minimize the surface area of polyethene required for the chosen cup.

$$
\frac{d A}{d r}=\frac{d}{d r}\left(\pi r^{2}+\pi(r+R) \sqrt{(r-R)^{2}+\left(\frac{3 V}{\pi\left(r^{2}+R r+R^{2}\right)}\right)^{2}}\right)
$$

Note that the root to equation 1.3 only provides the corresponding measurement for the bottom radius. To find the height, the obtained value is substituted for $r$ in equation 1.1. This then provides the optimal dimensions (i.e., the bottom radius and height) that minimize polyethene usage given a cup's volume and top radius.

\section{RESULTS}

To display its proper implementation, the strategy above is applied to the Starbucks Grande coffee cup. To start, a sample of ten Starbucks Grande coffee cups are randomly selected and their dimensions are measured. Table 1 summarizes the results of these measurements.

\begin{tabular}{|c|c|c|}
\hline Height (h) $\mathrm{cm}$ & Frequency & Midpoint $\mathrm{cm}$ \\
\hline $\mathbf{1 1 . 8}<\boldsymbol{h} \leq \mathbf{1 1 . 9}$ & 1 & 11.85 \\
\hline $\mathbf{1 1 . 9}<\boldsymbol{h} \leq \mathbf{1 2 . 0}$ & 6 & 11.95 \\
\hline $\mathbf{1 2 . 0}<\boldsymbol{h} \leq \mathbf{1 2 . 1}$ & 3 & 12.05 \\
\hline Top Diameter & Frequency & Midpoint $\mathrm{cm}$ \\
\hline (D) $\mathrm{cm}$ & & \\
\hline $\mathbf{8 . 3}<\boldsymbol{D} \leq \mathbf{8 . 4}$ & 6 & 8.35 \\
\hline $\mathbf{8 . 4}<\boldsymbol{D} \leq \mathbf{8 . 5}$ & 4 & 8.45 \\
\hline Bottom Diameter & Frequency & Midpoint $\mathrm{cm}$ \\
\hline $\mathbf{( d )} \boldsymbol{c m}$ & & \\
\hline $\mathbf{5 . 6}<\boldsymbol{d} \leq \mathbf{5 . 7}$ & 3 & 5.65 \\
\hline $\mathbf{5 .}<\boldsymbol{d} \leq \mathbf{5 . 8}$ & 7 & 5.75 \\
\hline
\end{tabular}

Table 1. Frequency table for the dimensions of a sample of Starbucks Grande coffee cups

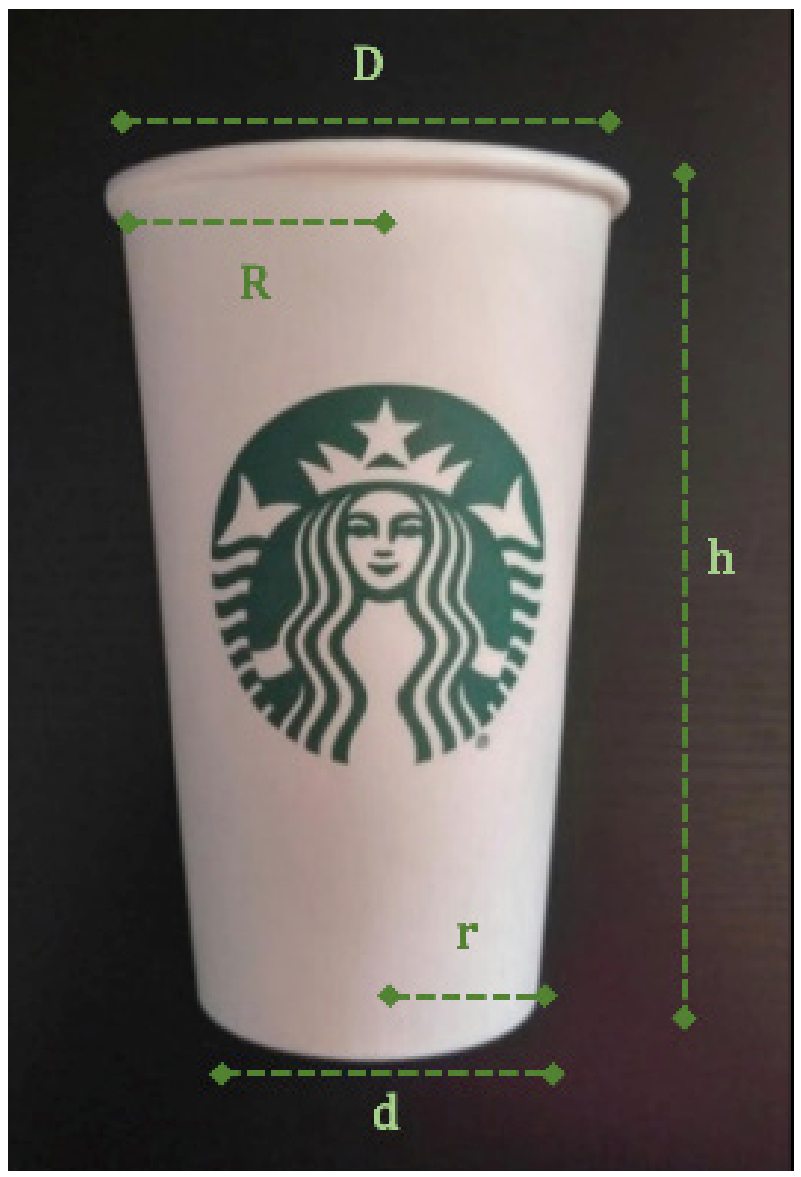

Figure 1. Starbucks Grande coffee cup 
The midpoints from each interval are used to evaluate the mean dimensions, with Table 2 summarizing the dimensions used for subsequent calculations in the section. Note that all measurements from this point moving forward are taken to three significant figures for consistency.

$$
\begin{gathered}
h=\frac{\sum_{i=1}^{k} f_{i} h_{i}}{n}=\frac{11.85 \times 1+11.95 \times 6+12.05 \times 3}{10}=12.0 \mathrm{~cm} \\
D=\frac{\sum_{i=1}^{k} f_{i} D_{i}}{n}=\frac{8.35 \times 6+8.45 \times 4}{10}=8.39 \mathrm{~cm} \\
d=\frac{\sum_{i=1}^{k} f_{i} d_{i}}{n}=\frac{5.65 \times 3+5.75 \times 7}{10}=5.72 \mathrm{~cm}
\end{gathered}
$$

\begin{tabular}{|c|c|c|c|c|}
\hline $\begin{array}{c}\text { Height } \\
\text { (h) }\end{array}$ & $\begin{array}{c}\text { Top Diameter } \\
\text { (D) }\end{array}$ & $\begin{array}{c}\text { Top Radius } \\
\text { (R) }\end{array}$ & $\begin{array}{c}\text { Bottom Diameter } \\
\text { (d) }\end{array}$ & $\begin{array}{c}\text { Bottom Radius } \\
(\mathbf{r})\end{array}$ \\
\hline $12.0 \mathrm{~cm}$ & $8.39 \mathrm{~cm}$ & $4.20 \mathrm{~cm}$ & $5.72 \mathrm{~cm}$ & $2.86 \mathrm{~cm}$ \\
\hline
\end{tabular}

Table 2. Mean dimensions for a Starbucks Grande coffee cup

Next, using the dimensions from Table 2 and the derived equation for the volume of a cup from Appendix $A$, the mean volume of the cup is evaluated.

$$
\begin{gathered}
V=\frac{\pi h}{3}\left(r^{2}+R r+R^{2}\right) \\
=\frac{\pi(12.0)}{3}\left[(2.86)^{2}+(4.20)(2.86)+(4.20)^{2}\right] \\
V=475 \mathrm{~cm}^{3}
\end{gathered}
$$

Lastly, the volume above and the dimensions from Table 2 are substituted into equation 1.3 as shown below. Using Desmos, the equation is graphed as shown in Figure 2, and its root is determined. Note that the domain of the graph is restricted to $r>0$ since $r$ represents a length and is consequently limited to positive numbers.

$$
\begin{gathered}
\frac{d A}{d r}=\frac{d}{d r}\left(\pi r^{2}+\pi(r+R) \sqrt{(r-R)^{2}+\left(\frac{3 V}{\pi\left(r^{2}+R r+R^{2}\right)}\right)^{2}}\right) \\
\frac{d A}{d r}=\frac{d}{d r}\left(\pi r^{2}+\pi(r+4.20) \sqrt{(r-4.20)^{2}+\left(\frac{1425}{\pi\left(r^{2}+4.20 r+4.20^{2}\right)}\right)^{2}}\right)
\end{gathered}
$$

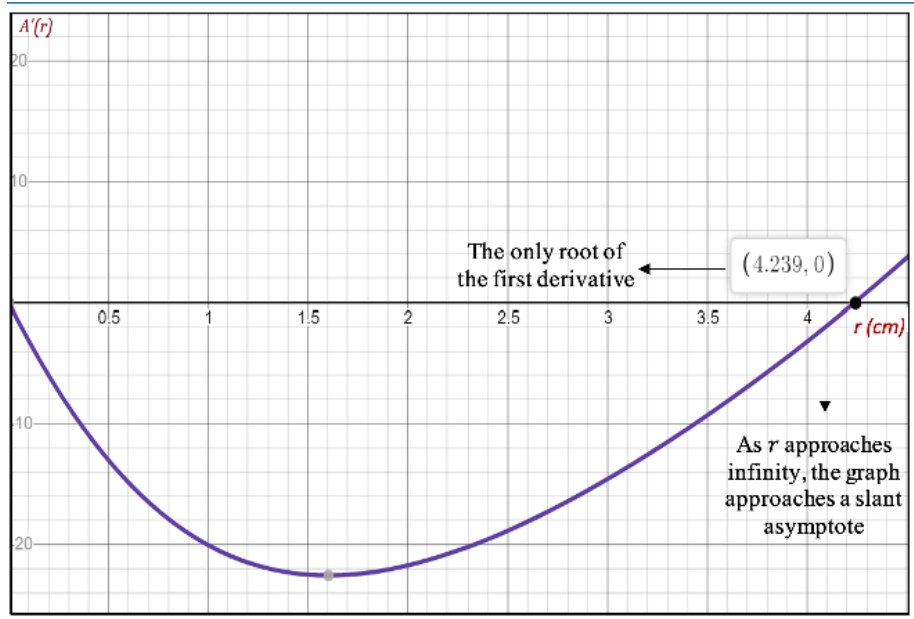

Figure 2. Graph for the first derivative of the surface area function

From Figure 2, it is observed that $r=4.24 \mathrm{~cm}$ is the only zero of the function, indicating that $\mathrm{r}=4.24 \mathrm{~cm}$ corresponds to either the minimum or maximum surface area. Since $r=4.24$ is preceded by negative values and is followed by positive values, $r=4.24$ must mathematically correspond to a minimum value.

Now, substituting $r=4.24$ into equation 1.1 determines the corresponding value of $h$.

$$
h=\frac{1425}{\pi\left(r^{2}+4.20 r+4.20^{2}\right)}=\frac{1425}{\pi\left(4.24^{2}+4.20 \times 4.24+4.20^{2}\right)}=8.49
$$

Thus, to minimize the amount of polyethene inside a Starbucks Grande coffee cup, the following dimensions are required for the bottom radius and height respectively:

$$
r=4.24 \mathrm{~cm} \quad h=8.49 \mathrm{~cm}
$$

\section{DISCUSSION}

Polyethene is an organic compound made from the polymerization of the ethene monomer, $\mathrm{C} 2 \mathrm{H} 4$. It is one of the most widely produced plastics due to its lightness, waterproof nature, and ability to retain heat, leading to its use inside paper coffee cups 


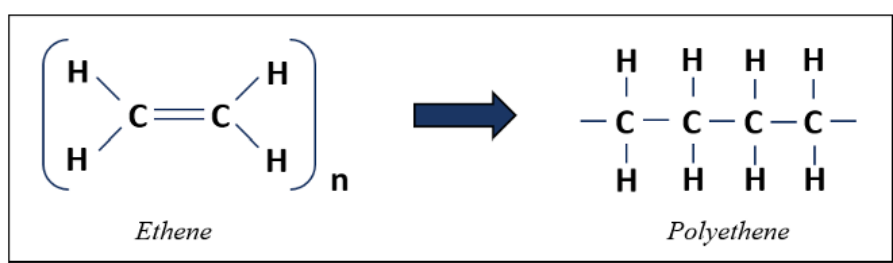

Figure 3. Structural formula for the polymerization of polyethene

Regardless of its benefits, the strong adhesion between polyethene and paper cups requires the use of complex and costly processing procedures, as well as specialized facilities to support coffee cup recycling (2). Thus, many recycling facilities tend to send paper coffee cups directly to landfills instead to avoid the extra costs (5). Within landfills, polyethene has a slow degradation rate due to the anaerobic environment, taking as long as 20 years to completely break down (6). This longevity results in the production of microplastics, which are defined as plastic products with dimensions less than $5 \mathrm{~mm}$ (7). These microplastics can enter the aquatic environment and where they are ingested by marine organisms. Once within the food chain, microplastics can proceed to negatively impact terrestrial organisms, including humans. Breakthrough studies as discussed by Sharma and Chatterjee have shown that microplastics can lead to the alteration of chromosomes and result in infertility, obesity, and cancer (7).

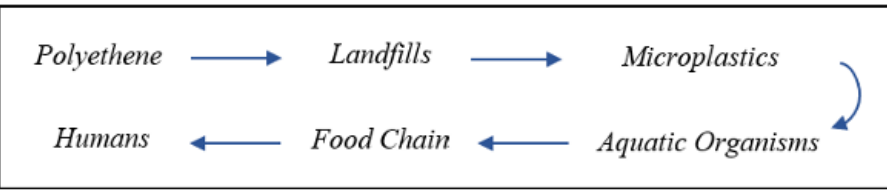

Figure 4. Life cycle of the polyethene inside paper coffee cups

Additionally, the usage of paper coffee cups on a global scale further escalates the severity of this problem. A recent study found that in 2016 alone, 269 billion disposable cups were used worldwide, with 146 billion of those cups belonging to North America (6). A long-term solution to the issue of the paper coffee cup requires a new design. Biodegradable cups, which use a corn-based plastic known as polylactic acid in place of polyethene, have been explored in the past as a potential alternative (8). However, these cups still require specific conditions for degradation, including high temperatures and long processing times (8). As a result, this design would still not circumvent the original problem of cups ending up in landfills. In response to this issue, some coffee companies have taken the initiative to reach out to the public for new innovations through coffee cup design challenges. Starbucks, which is estimated to have produced 3.85 billion paper cups in 2017 alone, launched its NextGen Cup Challenge in March 2019 and selected 12 prototypes for testing (9). The company's goal is to have its eco-friendly coffee cup developed by 2022, although no new progress has been made in recent time (9).

As the search for the 'perfect' design continues, most companies rely on two short-term strategies for reducing their polyethene production. The first approach focuses on public awareness and education, which has only resulted in minor behavioral changes in customers (2). The second approach is the promotion of reusable cups, which has led many local stores to create their own branded reusable cups, with Germany's Freiburg Cup and Australia's Keepcup as two well-known examples (10). On the other hand, the United Kingdom has been developing a tax for the use of paper coffee cups, a movement that has been estimated to increase the use of reusable coffee cups by only $3.4 \%$ (11). Additionally, to reach its 2008 goal of serving $25 \%$ of its drinks in reusable cups, Starbucks began offering $\$ 0.10$ discounts to customers who brought their own cups (12). However, the company abandoned this goal in 2018 after seeing that the movement resulted in less than $2 \%$ of its beverages being sold in reusable cups (4).

The main reason behind the limited success of the ongoing strategies is their dependence on customers who tend to choose the alternative that is most convenient to them rather than what is most environmentally friendly $(2,12)$. To overcome this barrier, this paper proposes a new strategy that allows for system-level changes. Using the derived formulae, companies can use their current cup dimensions to evaluate appropriate modifications to the height and bottom radius while keeping their volume and lid size the same. This will allow businesses to minimize the amount of polyethene used in each cup while the search for an eco-friendly design continues. Although the approach does not eliminate the use of polyethene, this reduction can still lead to a significant difference considering the large number of coffee cups sent to landfills annually and the uncertainty around when the ideal cup design will be available.

Nonetheless, regardless of its benefits, it is important to consider the limitations underlying the application of this method, as highlighted for the results of the Starbucks Grande coffee cup. Firstly, the sample size was limited to 10 coffee 
cups to minimize the amount of waste produced. Secondly, the procedure was limited to three significant figures for all calculations to maintain consistency. Thirdly, the optimal design was limited to the standard conical frustum and did not consider a complete change in the design. However, this raises an interesting question worthy of additional investigation: what if the design of the cup was to be fully re-developed? Such an extension would compare cups of different geometric shapes to see which uses the least amount of polyethylene.

Additionally, it is important to acknowledge that the obtained results only provide an approximation for the optimal dimensions of an eco-friendly cup due to the presumed assumptions summarized in Table 3.

\begin{tabular}{|l|l|}
\hline \multicolumn{1}{|c|}{ Assumption } & \multicolumn{1}{|c|}{ Impact } \\
\hline $\begin{array}{l}\text { 1. The cup's internal surface area equals the } \\
\text { surface area of polyethylene. }\end{array}$ & $\begin{array}{l}\text { More polyethylene is most likely required } \\
\text { to fully secure the coating on the cup. }\end{array}$ \\
\hline $\begin{array}{l}\text { 2. The width of polyethylene is negligible. This } \\
\text { assumption was made due to the absence of } \\
\text { information on the width of the polyethylene } \\
\text { coating present inside paper coffee cups. }\end{array}$ & $\begin{array}{l}\text { The width of coating influences the } \\
\text { amount of polyethylene required. }\end{array}$ \\
\hline $\begin{array}{l}\text { 3. The paper coffee cup takes the form of a } \\
\text { perfect conical frustum. }\end{array}$ & $\begin{array}{l}\text { The top of the cup is slightly curved, } \\
\text { implying that more polyethylene may be } \\
\text { required than assumed. }\end{array}$ \\
\hline
\end{tabular}

Table 3 Assumptions made for the investigation and their corresponding impacts

\section{CONCLUSION}

Contrary to popular belief, many paper coffee cups end up in landfills due to the polyethene coating covering their internal surface area and break down into microplastics (1, 2). Once present within the environment, microplastics are ingested by organisms and enter the food chain where they can go on to impact the health of numerous other organisms $(2,3)$. As a result, many companies around the world have begun searching for an eco-friendly cup design while also implementing temporary strategies to reduce polyethene production $(9,11)$. However, many of such short-term strategies produce limited success due to their dependence on customer collaboration (2).

One strategy that is independent of customer cooperation is to adjust coffee cup dimensions to minimize the amount of polyethene required for the internal surface area. This method treats the volume and top radius of the original cup as constants and thus provides the optimal dimensions for the height and bottom radius. To apply this method, the me- asurements for the current volume and top radius are substituted into the equation below. Note that $\mathrm{V}$ and $\mathrm{R}$ are not variables but constants.

$$
\frac{d A}{d r}=\frac{d}{d r}\left(\pi r^{2}+\pi(r+R) \sqrt{(r-R)^{2}+\left(\frac{3 V}{\pi\left(r^{2}+R r+R^{2}\right)}\right)^{2}}\right)
$$

where:

$$
\begin{aligned}
& V=\text { volume of conical frustum } \quad r=\text { bottom radius of conical frustum } \\
& R=\text { top radius of conical frustum }
\end{aligned}
$$

Then, using technology, the root of the equation and hence the optimal dimension for the bottom radius is determined. Lastly, using the provided equation and the determined bottom radius, the optimal height is also evaluated.

$$
h=\frac{3 V}{\pi\left(r^{2}+R r+R^{2}\right)}
$$

As a result of its sole reliance on the company and not the customers, this strategy will allow a reduction in polyethene production while the search for an alternative cup design continues. Companies can use this method to find the optimal dimensions for their various cup sizes until a design that fully eliminates the use of polyethene is developed.

\section{REFERENCES}

1. Gabbatiss J. The UK throws away 2.5 billion disposable coffee cups every year [Internet]. Independent.co.uk. 2018 [cited 27 June 2020]. Available from: https://www. independent.co.uk/environment/disposable-coffeecups-how-big-problem-environment-landfill-recyclingincinerate-export-rubbish-a8142381.html

2. Yuhui M. Problems and Resolutions in Dealing with Waste Disposable Paper Cups. Sci Prog. 2018;101(1):1-7.

3. Kalogerakis N, Karkanorachaki K, Kalogerakis G, Triantafyllidi E, Gotsis A, Partsinevelos P et al. Microplastics Generation: Onset of Fragmentation of Polyethylene Films in Marine Environment Mesocosms. Front Mar Sci. 2017;4:1-15.

4. Lawley J. Fogg Model of Behavior Change [Internet]. Cleanlanguage.co.uk. 2013 [cited 27 June 2020]. Available from: https://www.cleanlanguage.co.uk/articles/ blogs/91/

5. Grover A, Gupta A, Chandra S, Kumari A, Khurana P. Polythene and environment. Int J Environ Sci. 2015;5(6):1091-1105.

6. BASF. The anatomy of a cup [Internet]. BASF. 2017 [cited 27 June 2020]. Available from: https://insights.basf.com/ home/article/read/anatomy-of-a-cup

7. Sharma S, Chatterjee S. Microplastic pollution, a threat 
to marine ecosystem and human health: a short review. Environ Sci Pollut Res Int. 2017;24(27):21530-21547.

8. Ziada H. Disposable Coffee Cup Waste Reduction Study [Internet]. McMaster University Faculty of Engineering; 2009 [cited 27 June 2020]. Available from: https://www. eng.mcmaster.ca/sites/default/files/uploads/disposable_ coffe_cup_waste_reduction.pdf

9. Lyons K. Starbucks begins in-store testing of greener cup [Internet]. The Verge. 2020 [cited 27 June 2020]. Available from: https://www.theverge.com/2020/3/9/21171364/ starbucks-new-cup-biodegradable-liner-recyclesustainable-test

10. Toor S, Khan M, Dhir N, Bajwa A. Ecological and social costs of single use coffee cups [Internet]. The University of British Columbia. 2018 [cited 27 June 2020]. Available from: https://cases.open.ubc.ca/w17t2con200-3/

11. Smithers R. Reusable incentives could slash disposable coffee cup waste [Internet]. The Guardian. 2017 [cited 27 June 2020]. Available from: https://www.theguardian. com/environment/2017/mar/30/reusable-incentivescould-slash-disposable-coffee-cup-waste

12. Lee J. A study for increasing reusable cup consumption in the coffee industry: Focused on behavior change with motivation [Master's thesis on the Internet]. lowa State University; 2015 [cited 27 June 2020]. Available from: https://lib.dr.iastate.edu/etd/14847/

\section{APPENDICES}

Appendix A - Deriving a Formula for the Volume of a Coffee Cup

Consider the cup created by rotating the enclosed region in Figure 5 around the $x$-axis. By vertically slicing the cup into thin pieces, an infinite number of thin cylindrical disks will be created, each with an area of $\pi[f(x)]^{\wedge} 2$. The volume of the cup is then found by summing the areas of all these disks, where $f(x)$ represents the cup's slanted length.

$V=\lim _{n \rightarrow \infty} \sum_{i=1}^{n} f\left(x_{i}\right)\left(x_{i+1}-x_{i}\right)=\int_{0}^{h} \pi[\boldsymbol{f}(x)]^{2} \boldsymbol{d} \boldsymbol{x}$

sing the slope-intercept form, $f(x)$ is rewritten.

$$
\begin{gathered}
m=\frac{y_{2}-y_{1}}{x_{2}-x_{1}}=\frac{r-R}{h} \\
f(x)=m x+b=\frac{\boldsymbol{r}-\boldsymbol{R}}{\boldsymbol{h}} \boldsymbol{x}+\boldsymbol{R}
\end{gathered}
$$

Next, equation 1.5 is substituted for $f(x)$ in equation 1.4. This allows the definite integral from equation 1.4 to be eva- luated, which provides an equation tor volume that is expressed in terms of the cup's height, bottom radius, and top radius.

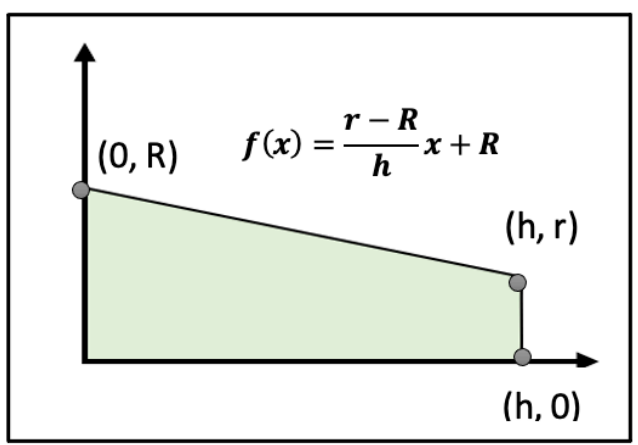

Figure 5. An enclosed region

$$
\begin{gathered}
V=\int_{0}^{h} \pi[f(x)]^{2} d x \\
=\int_{0}^{h} \pi\left(\frac{r-R}{h} x+R\right)^{2} d x \\
=\pi \int_{0}^{h}\left(\frac{(r-R)^{2}}{h^{2}} x^{2}+2 R \frac{r-R}{h} x+R^{2}\right) d x \\
\left.=\pi \Gamma^{(r-R)^{2}} x^{3}+R{ }^{r-R} x^{2}+R^{2} x\right]_{b}^{R} \\
=\pi\left[\frac{(r-R)^{2}}{3 h^{2}} h^{3}+R \frac{r-R}{h} h^{2}+R^{2} h\right]-\pi\left[\frac{(r-R)^{2}}{3 h^{2}}(0)^{3}+R \frac{r-k}{h}(0)^{2}+R^{2}(0)\right] \\
\left.=\pi\left[\begin{array}{c}
h(r-R)^{2} \\
3
\end{array}\right]+R h(r-R)+R^{2} h\right] \\
=\pi \frac{h(r-R)^{2}+3 R h(r-R)+3 R^{2} h}{3} \\
h r^{2}-2 h R r+h R^{2}+3 h R r-3 R^{2} h+3 R^{2} h \\
3 \\
=\pi \frac{h r^{2}+h R r+h R^{2}}{3} \\
V=\frac{\pi h}{3}\left(r^{2}+R r+R^{2}\right)
\end{gathered}
$$

where:

$$
\begin{array}{ll}
V=\text { volume of conical fruslum } & r=\text { bottom radius ol conical frustum } \\
R=\text { top radius of conical frustum } & h=\text { height of conical frustum }
\end{array}
$$

Appendix B - Deriving a Formula for the Surface Area of a Coffee Cup

Before determining a formula for the surface area of a coffee cup, a more general formula for the surface area of a solid of revolution is obtained.

Consider the cone in Figure 6, with its lateral surface flattened. Since the arc of the lateral surface is lined up along the circumference of the circular base, its length equals $2 \pi r$. 
Rearranging the equation for arc length expresses angle $\theta$ in terms of $s$ and $r$.

$$
\begin{gathered}
l=\theta R \\
\theta=\frac{l}{R}=\frac{2 \pi r}{s}
\end{gathered}
$$

The area of the lateral surface is then rewritten.

$$
A=\frac{1}{2} \theta r^{2}=\frac{1}{2} \times \frac{2 \pi r}{s} \times s^{2}=\pi r s
$$

where:

$$
\begin{aligned}
& r=\text { radius of the cone's base } \\
& s=\text { slant length }
\end{aligned}
$$

The surface area of a solid of revolution is approximated by breaking down the solid into smaller bands in the form of conical frustums and then summing the areas of the frustums' lateral surfaces. Consider the frustum in Figure 7, which is extended to form a cone. The lateral area of the frustum is obtained by subtracting the lateral area of the small cone from the lateral area of the large cone using equation 1.6

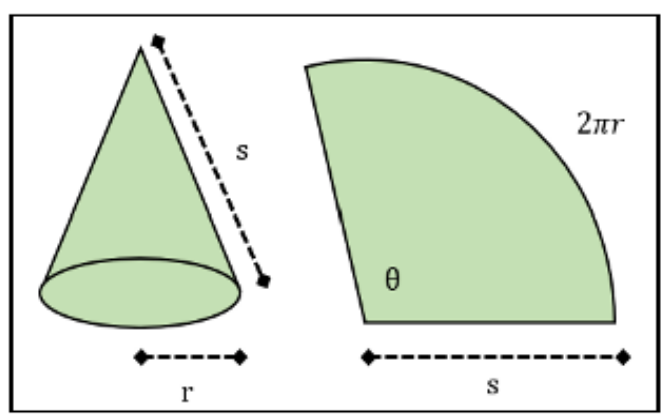

Figure 6. The lateral surface of a cone

$$
\begin{gathered}
A=\pi r_{2} s_{2}-\pi r_{1} s_{1}=\pi r_{2}\left(l_{2}+l_{1}\right)-\pi r_{1} l_{1} \\
A=\pi\left[r_{2} l_{2}+r_{2} l_{1}-r_{1} l_{1}\right]
\end{gathered}
$$

By triangle similarity, the following must be true.

$$
\begin{gathered}
\frac{l_{1}+l_{2}}{r_{2}}=\frac{l_{1}}{r_{1}} \\
r_{2} l_{1}=r_{1} l_{1}+r_{1} l_{2}
\end{gathered}
$$

Substituting equation 1.8 into 1.7 provides a new equation for the lateral area of a frustum

$$
A=\pi\left[r_{2} l_{2}+r_{2} l_{1}-r_{1} l_{1}\right]=\pi\left(r_{2} l_{2}+r_{1} l_{1}+r_{1} l_{2}-r_{1} l_{1}\right)=\pi\left(r_{1}+r_{2}\right) l_{2}
$$

Note that the above equation is not used to find the cup's surface area since it does not express the lateral surface area in terms of $h$. Instead, the process is further carried out by rewriting equation 1.9 as followed.

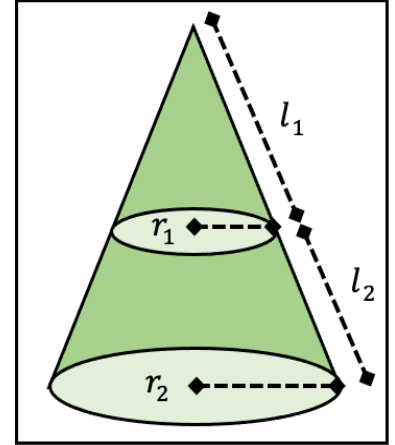

Figure 7. Relationship between a frustum and a cone

$$
A=\pi\left(r_{1}+r_{2}\right) l=2 \pi r l
$$

where:

$$
\begin{aligned}
& r=\frac{1}{2}\left(r_{1}+r_{2}\right)=\text { average radius of frustum } \\
& l=\text { slant length of frustum }
\end{aligned}
$$

Now, consider the case where the solid of revolution is broken down into small frustums. For each frustum, the endpoints of the slant length on a two-dimensional plane have coordinates ( $\left.x_{-}(i) f,\left(x_{-} i\right)\right)$ and $\left(x_{-}(i-1) f,\left(x_{-}(i-1)\right)\right)$, as shown in Figure 8 . The slant length is then determined using the mathematical equation for the distance between two points.

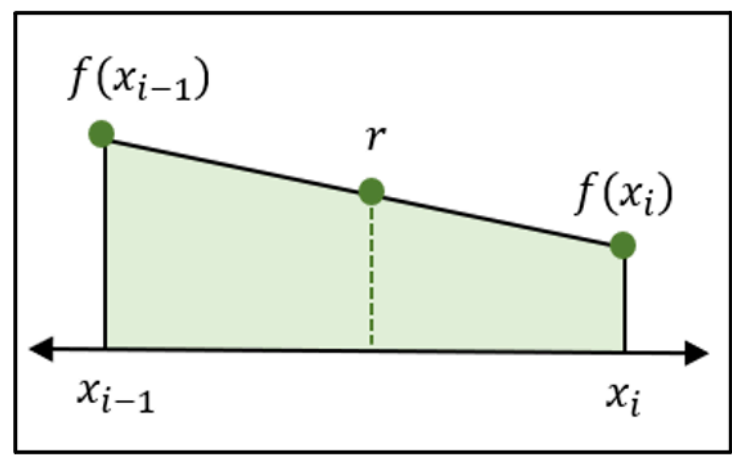

Figure 8. Average radius of a frustum

$$
\begin{gathered}
d=\sqrt{\left(x_{1}-x_{2}\right)^{2}+\left(y_{1}-y_{2}\right)^{2}} \\
l=\sqrt{\left(x_{i}-x_{i-1}\right)^{2}+\left(f\left(x_{i}\right)-f\left(x_{i-1}\right)\right)^{2}}=\sqrt{\Delta \boldsymbol{x}^{2}+\Delta \boldsymbol{f}\left(\boldsymbol{x}_{\boldsymbol{i}}\right)^{2}}
\end{gathered}
$$

By the Mean Value Theorem, on the continuous and differentiable interval $\left[x_{-}(i-1), x_{-}(i),\right]$, there must be a point $x_{-}{ }^{\wedge}{ }^{\wedge}$ such that 


$$
\begin{gathered}
f\left(x_{i,}\right)-f\left(x_{i-1}\right)=f^{\prime}\left(x_{i}^{*}\right)\left(x_{i}-x_{i-1}\right) \\
\Delta f\left(x_{i}\right)=\boldsymbol{f}^{\prime}\left(\boldsymbol{x}_{i}^{*}\right) \Delta x
\end{gathered}
$$

Substituting equation 2.3 into 2.2 then provides the following:

$$
l=\sqrt{\Delta x^{2}+\Delta f\left(x_{i}\right)^{2}}=\sqrt{\Delta x^{2}+\left(f^{\prime}\left(x_{i}^{*}\right) \Delta x\right)^{2}}=\sqrt{\mathbf{1}+\left(f^{\prime}\left(\boldsymbol{x}_{i}^{*}\right)\right)^{2}} \Delta \boldsymbol{x}
$$

Finally, equation 2.4 is substituted for $l$ in equation 2.1 as shown.

$$
A=2 \pi r l=2 \pi r \sqrt{1+\left(f^{\prime}\left(x_{i}^{*}\right)\right)^{2}} \Delta x
$$

As highlighted in Figure 4, since $r$ is the average radius of the frustum:

$$
r=\frac{f\left(x_{i-1}\right)+f\left(x_{i}\right)}{2}
$$

Considering that the original solid of revolution has been broken down into many small frustums, $\Delta x$ must be very small. Thus, it can be assumed that $\mathrm{f}\left(\mathrm{x}_{-}(\mathrm{i}-1)\right) \approx \mathrm{f}\left(\mathrm{x}_{-} \mathrm{i} \wedge *\right.$ ) and $f\left(x_{-} i\right) \approx f\left(x_{-} i^{\wedge}\right)$, which allows $r$ to be expressed as shown:

$$
r \approx \frac{f\left(x_{i}^{*}\right)+f\left(x_{i}^{*}\right)}{2}=f\left(x_{i}^{*}\right)
$$

Hence, an approximate area for the solid of revolution is obtained by breaking down the solid into $\mathrm{n}$ frustums and summing all their areas.

$$
A \approx \sum_{i=1}^{n} 2 \pi f\left(x_{i}^{*}\right) \sqrt{1+\left(f^{\prime}\left(x_{i}^{*}\right)\right)^{2}} \Delta x
$$

Since the approximation becomes more accurate as $n->\infty$, an exact equation can be obtained for the solid's area.

$$
A=\lim _{n \rightarrow \infty} \sum_{i=1}^{n} 2 \pi f\left(x_{i}^{*}\right) \sqrt{1+\left(f^{\prime}\left(x_{i}^{*}\right)\right)^{2}} \Delta x=\int_{a}^{b} 2 \pi f(x) \sqrt{1+\left(f^{\prime}(x)\right)^{2}} d x
$$

where:

$f(x)=$ slant length of frustum

Finally, to find a formula for the surface area of a coffee cup, equation 1.5 is substituted for $f(x)$ in equation 2.5 and is then simplified.

$$
\begin{aligned}
& A=\pi r^{2}+\int_{a}^{b} 2 \pi f(x) \sqrt{1+\left(f^{\prime}(x)\right)^{2}} d x \\
& =\pi r^{2}+\int_{0}^{h} 2 \pi\left({ }_{h}^{r-R} x+R\right) \sqrt{1+\left(\frac{d}{d x}\left(\begin{array}{r}
r-R \\
h
\end{array}+R\right)\right)^{2}} d x \\
& =\pi r^{2}+\int_{0}^{h} 2 \pi\left(\frac{r-R}{h} x+R\right) \sqrt{1+\left(\frac{r-R}{h}\right)^{2}} d x \\
& =\pi r^{2}+\int_{0}^{h} 2 \pi\left({ }_{h}^{r-R} x+R\right) \sqrt{\frac{(r-R)^{2}+h^{2}}{h^{2}}} d x \\
& =\pi r^{2}+\frac{2 \pi}{h} \sqrt{(r-R)^{2}+h^{2}} \int_{0}^{h}\left(\frac{r-R}{h} x+R\right) d x \\
& =\pi r^{2}+\frac{2 \pi}{h} \sqrt{(r-R)^{2}+h^{2}}\left[\frac{r-R}{2 h} x^{2}+R x\right]_{0}^{h} \\
& =\pi r^{2}+\frac{2 \pi}{h} \sqrt{(r-R)^{2}+h^{2}}\left(\frac{(r-R) h}{2}+R h\right) \\
& =\pi r^{2}+\frac{2 i u}{h} \sqrt{(r-R)^{2}+h^{2}}\left(\frac{r h-R h_{2}+2 R h}{2}\right) \\
& =\pi r^{2}+\frac{2 \pi}{h} \sqrt{(r-R)^{2}+h^{2}}\left(\frac{h(r+k)}{2}\right) \\
& =\pi r^{2}+2 \pi \sqrt{(r-R)^{2}+h^{2}}\left(\frac{r+R}{2}\right) \\
& A=\pi r^{2}+\pi(r+R) \sqrt{(r-R)^{2}+h^{2}}
\end{aligned}
$$

where:

$$
\begin{array}{ll}
A=\text { surlace area of cup } & h=\text { height ol cup } \\
R=\text { top radius of cup } & r=\text { bottom radius of cup }
\end{array}
$$




\section{Viewpoints}

